\title{
Experimental Comparison of Probabilistic Shaping with online PMF Optimization and Mid-link OPC
}

Yankov, Metodi Plamenov; Da Ros, Francesco; Porto da Silva, Edson; Galili, Michael; Oxenløwe, Leif Katsuo

Published in:

CLEO: Science and Innovations 2018

Link to article, DOI:

10.1364/CLEO_SI.2018.STu4C.2

Publication date:

2018

Document Version

Peer reviewed version

Link back to DTU Orbit

Citation (APA):

Yankov, M. P., Da Ros, F., Porto da Silva, E., Galili, M., \& Oxenløwe, L. K. (2018). Experimental Comparison of Probabilistic Shaping with online PMF Optimization and Mid-link OPC. In CLEO: Science and Innovations 2018 [Paper STu4C.2] Optical Society of America. https://doi.org/10.1364/CLEO_SI.2018.STu4C.2

\section{General rights}

Copyright and moral rights for the publications made accessible in the public portal are retained by the authors and/or other copyright owners and it is a condition of accessing publications that users recognise and abide by the legal requirements associated with these rights.

- Users may download and print one copy of any publication from the public portal for the purpose of private study or research.

- You may not further distribute the material or use it for any profit-making activity or commercial gain

- You may freely distribute the URL identifying the publication in the public portal 


\title{
Experimental Comparison of Probabilistic Shaping with online PMF Optimization and Mid-link OPC
}

\author{
M.P. Yankov ${ }^{1,2}$, F. Da Ros ${ }^{1}$, E. P.da Silva ${ }^{1}$, M. Galili ${ }^{1}$, and L.K. Oxenløwe ${ }^{1}$ \\ 1 DTU Fotonik, Technical University of Denmark, DK-2800 Kongens Lyngby, Denmark, fdro@fotonik.dtu.dk \\ 2 Fingerprint Cards ApS, Lyskarr 3CD, 2730 Herlev, Denmark, meya@fotonik.dtu.dk
}

\begin{abstract}
Gains offered by mid-link OPC and probabilistic shaping are compared in a dispersionmanaged link with 64QAM input. Probabilistic shaping is optimized online and tailored to the specific channel and transceiver conditions, including the OPC stage.

OCIS codes: (060.1660) Coherent communications, (060.0060) Fiber optics and optical communications,
\end{abstract}

\section{Introduction}

Nonlinearity mitigation and compensation techniques have attracted significant interest in the optical fiber communications community as tools to increase the data rates of standard, single mode fiber (SSMF) links. Optical phase conjugation (OPC) [1] is a promising optical technique since it allows for full-band optical nonlinear compensation. Probabilistic shaping (PS) is a digital tool used for improving data rates, by increasing the tolerance of the signal to the linear noise due to amplification [2-4]. Probabilistic shaping entails optimization of the probability mass function (PMF) of the input signal. This optimization can be efficiently done e.g. with a modified Blahut-Arimoto algorithm (BAA) as in [3] which also allows for tailoring the PMF to the specific channel of interest. In this paper, the BAA is applied to a dispersion managed channel with and without mid-link OPC (MLOPC) for a 64 quadrature amplitude modulation (QAM) input. Therefore, the channel of interest for PS includes the OPC stage, effectively combining digital and optical techniques. The PMF is directly optimized on the experimental setup, and is thus tailored to all practical imperfections present in the link, e.g. optical modulation imperfections, DSP imperfections, digital-to-analog and analog-to-digital conversion (DAC and ADC, respectively) quantization noise, etc. The gains from the optical (OPC) and digital (PS) techniques are then compared and their relation is analyzed.

\section{Experimental setup}

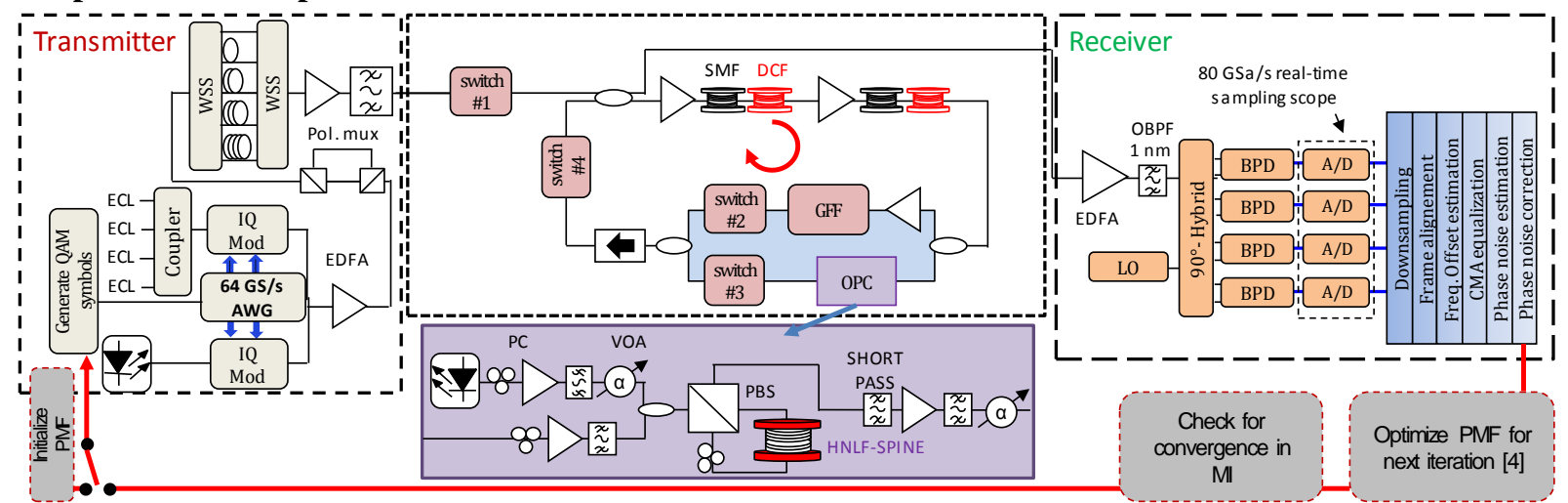

Fig. 1. Experimental setup for the transmission measurements comparing straight transmission to OPC-based transmission. Insets: i) schematic structure of the waveguide with lateral p-i-n diode and ii) optical spectra (red) and CW CE (blue) at port 3 of the circulator in the OPC.

The experimental setup is shown in Fig. 1. The transmitter consists of 5 external cavity lasers (ECL, 10-kHz linewidth, $25 \mathrm{GHz}$ spacing) modulated by two IQ modulators driven by an arbitrary waveform generator (AWG). The 64QAM data symbols are interleaved with 4QAM pilots (pilot rate of 10\%) used for equalization [6], and loaded in the AWG at $16 \mathrm{GBd}$. Square root raised cosine (RRC) pulse-shaping is applied with a roll-off factor of 0.01 . The channels are combined, a delay-and-add polarization emulator generates a dual-polarization signal and a fiber-based decorrelation stage based on two wavelength selective switches (WSS) provides full decorrelation (> 70 symbols) of the wavelength division multiplexed (WDM) channels. The five channels are then launched into a recirculating transmission loop (total length of $140 \mathrm{~km}$ ) composed of two dispersion-compensated spans (90 and 50 $\mathrm{km}$ ) and erbium doped fiber amplifiers (EDFAs). Inside the loop, acousto-optic switches select either the straight path (switch \#2) consisting of an EDFA and a gain flattening filter (GFF), or the OPC path (switch \#3). The OPC is based on a single-pump $\left(\lambda_{\mathrm{p}}=1545 \mathrm{~nm}\right)$ four-wave mixing stage in a polarization-diversity loop with a 500-m long highly nonlinear fiber (OFS, HNLF-SPINE) as in [7]. The conversion efficiency is $-17 \mathrm{~dB}$ as shown in Fig. 2(a). 
At the output of the OPC, only the idler band is selected for further propagation. After transmission, the signal is received with a pre-amplified coherent receiver based on a digital storage oscilloscope (DSO). Offline processing follows, including down sampling, frequency offset estimation based on the pilots, time-domain equalization using a pilot-based constant modulus algorithm (CMA) [6] and carrier phase recovery [3].

The iterative BAA used for PMF optimization relies on the received samples after the DSP chain to maximize mutual information (MI) and provide a new PMF for the next iteration. This approach ensures that the PMF is optimized for the particular channel conditions where the 'channel' includes all components and signal processing between the transmitted QAM symbols and the noisy received QAM symbols, i.e. pulse shaping, DAC, optical modulation, fiber transmission, optical front end at the receiver, ADC and DSP. The MI is estimated based on the mismatched decoding principle with circular Gaussian auxiliary channel [3]. An illustration of the optimization procedure is given in the bottom part of Fig. 1 and the iterative process is stopped upon convergence of the MI with a convergence threshold chosen as when the MI improvement is less than $0.01 \mathrm{bits} / \mathrm{symbol}$.

\section{Transmission results}

The spectra of the signals before and after the OPC stage are given in Fig. 2(a) and the received effective signal-tonoise ratio (SNR) is shown in Fig. 2(b) for both straight and ML-OPC cases. The effective SNR is estimated from the transmitted and received QAM symbols as in [4] and is similar for uniform and PS signaling. As shown, ML-OPC provides a gain of approx. $0.6 \mathrm{~dB}$ and shifts the optimum launched power by $2 \mathrm{~dB}$. Finally, Fig. 2(c) shows the MI for the four scenarios considered. ML-OPC and PS both achieve gains but from different means: ML-OPC aims at reducing the nonlinear interference noise (NLIN) variance, while PS aims at optimizing the transmission for a given total noise variance (effective SNR). The optimal launch power with ML-OPC is thus increased, while with PS, the system operates at lower launch power, for which the noise is more Gaussian and independent, identically distributed in time. For the considered setup, ML-OPC provides approx. $0.19 \mathrm{bit} / \mathrm{symbol}$ of gain, and PS approx. $0.3 \mathrm{bits} / \mathrm{symbol}$ for both cases of straight and ML-OPC transmission. These results show that the gains of the two techniques add-up. To the extent of this analysis, the two techniques are therefore independent, and even though the optimal launch power is different, the NLIN statistics of ML-OPC and standard links at the optimal launch power, are qualitatively similar.
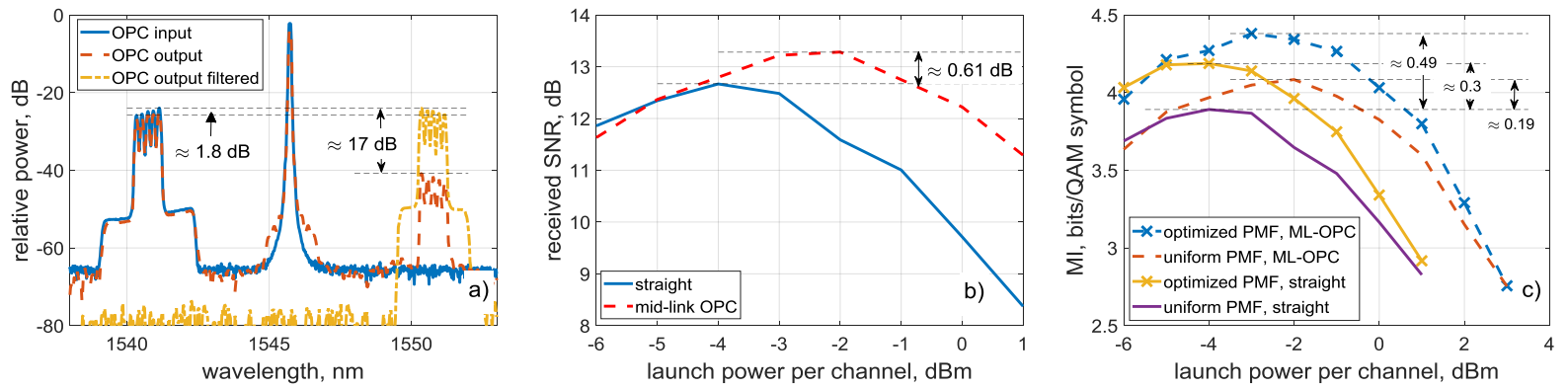

Fig. 2. Performance of straight and ML-OPC transmission. a): Spectra before and after the OPC stage; b): Received effective SNR (identical with and without PS; c): MI with and without PS. The gains from ML-OPC and PS appear independent and to add-up.

\section{Conclusions}

The gains of probabilistic shaping and mid-link OPC are experimentally demonstrated to be independent and to add up in a dispersion managed EDFA based link. Combination of both techniques is therefore of interest for future fiber optic networks aiming at both compensating nonlinearity and operate as close as possible to channel capacity.

\section{Acknowledgements}

This work was supported by the DFF project NANO-SPECs (DFF-4005-00558B), and the DNRF Research CoE, SPOC (ref. DNRF123).

\section{References}

[1] A.D. Ellis, et al., " $4 \mathrm{~Tb} / \mathrm{s}$ transmission reach enhancement using $10 \times 400 \mathrm{~Gb} / \mathrm{s}$ super-channels and polarization insensitive dual band optical phase conjugation," J. of Lightw. Technol. 34(8), 1717-1723 (2016).

[2] F. Buchali, et al., "Rate adaptation and reach increase by probabilistically shaped 64- QAM: An experimental demonstration," J. of Lightw. Technol. 34(7), 1599-1609 (2016).

[3] M.P. Yankov et al., "Constellation shaping for WDM systems using 256QAM/1024QAM with probabilistic optimization,” J. of Lightw. Technol. 34(22), 5146-5156 (2016).

[4] T. Fehenberger et al., "On probabilistic shaping of quadrature amplitude modulation for the nonlinear fiber channel," J. of Lightw. Technol. 34(21), 5063-5073 (2016).

[5] S. Verdú and Y. Wu, "Functional properties of minimum mean-square error and mutual information," IEEE Trans. on Inf. Theory, 58(3), 1289-1301, 2012.

[6] M. P. Yankov et al., "Experimental analysis of pilot-based equalization for probabilistically shaped WDM systems with 256QAM/1024QAM," in Proc. of OFC 2017, paper W.2.A.48.

[7] F. Da Ros et al., "Impact of signal-conjugate wavelength shift on optical phase conjugation-based transmission of QAM signals," in Proc. of ECOC 2017, paper P1.SC4.66. 